\title{
Flow Behavior of Oil-in-Water Emulsions
}

\author{
Yasufumi Otsubo*1 and Robert K. Prud'HOMmE*2 \\ ${ }^{*}{ }^{1}$ Department of Image Science, Faculty of Engineering, Chiba University, \\ Yayoi-cho 1-33, Chiba-shi, 260, Japan \\ *2Department of Chemical Engineering, Princeton University, \\ Princeton, New Jersey 08544, U.S.A.
}

(Received: November, 6, 1991)

\begin{abstract}
The flow behavior of oil-in-water emulsions is examined as a function of the volume fraction of dispersed drops, drop size, and viscosity ratio of internal to external phases. The results are interpretted in terms of internal circulation, deformation, and breakup of drops. At low volume fractions, the viscosity decreases with increasing shear rate and becomes constant at high shear rates. The Newtonian viscosity is independent of drop size. The viscosity in the pseudoplastic region increases with decreasing drop size. A change in drop size at constant volume fraction causes a horizontal shift in the viscosity versus shear rate curve. As the viscosity ratio decreases, the relative viscosity at a given volume fraction is reduced because hydrodynamic forces cause internal circulation which reduces the velocity perturbations outside drops. At high volume fractions where a network of thin liquid films is formed, the change in drop size leads to a vertical shift of the viscosity curve. The flow behavior in highly concentrated emulsions is governed by total interfacial area. When the deformation and breakup of drops occur at high shear rates, the emulsions show pseudoplastic flow. Key words: Oil-in-water emulsion / Flow behavior / Internal circulation / Drop deformation / Interfacial area
\end{abstract}

\section{Introduction}

Liquid drops suspended in an immiscible liquid show three types of dynamic responses to shear fields: internal circulation, deformation and orientation, and drop breakup or coalescence. These effects are not observed for rigid spheres and hence the rheological properties of emulsions are different from those of suspensions. The most significant difference is that highly concentrated emulsions show elastic behavior at small strains resulting from the interfacial energy associated with deformation of thin lamellae between the dispersed phase drops. When the liquid films or lamellae are very thin, interfacial tension is primarily responsible for the solid-like behavior such as a static shear modulus and plastic yield. The first picture of elasticity and yielding behavior was given by Princen $^{1) 3 \text { ) }}$ and Prud'homme $e^{4}$ on the basis of a dry foam model in which the only forces acting on a cell are the interfacial tension along each liquid film. This model predicts that both the yield stress and shear modulus are proportional to the interfacial tension and inversely proportional to the drop radius. When the applied stress exceeds the yield stress, the emulsion starts to flow. Assuming that the original network structures of cells are periodically generated through coalescense and disproportionation processes during flow, the steady-flow behavior can be predicted from the shear stress as a function of $\operatorname{strain}^{5), 6)}$. However, the shear rate effect is not provided by the previous model because the viscous forces are neglected. Recently, Khan and Armstrong ${ }^{6) \sim 8}$ ) have extended the dry foam model by considering the viscosity of continuous phase and predicted the shear thinning behavior of foams.

On the other hand, when the volume fraction of drops is low, the theories for dilute suspensions can be used to describe the rheology. Taylor ${ }^{9}$ extended Einstein's equation to include spherical liquid drops by taking into account the internal circulation in the drops. Since the circulation inside the drops reduces the distortion of the velocity field outside the drops, the viscosity of emulsions decreases with decreasing viscosity of internal phase. Taylor's theory assumes that the drops remain spherical, although in fact they tend to be distorted into ellipsoids in shear fields. The drop deformation and orientation are complicated even in hydrodynamically isolated emulsions ${ }^{10) \sim 12)}$, so it is very difficult to theoretically derive a viscosity equation valid for 
moderately concentrated emulsions ${ }^{13), 14)}$.

There are many experimental studies of emulsion rheology ${ }^{15}$ )18), but the subject is still not well understood because of the lack of comprehensive and systematic data. This is related to difficulties in producing stable, well-characterized emulsions. Methods to prepare stable emulsions up to a volume fraction of 0.95 have been reported by several authors ${ }^{2), 19) \sim 21)}$. Such stable emulsions enable us to obtain rheological information over a wide range of concentration. The viscosity behavior of oil-in-water emulsions has been measured as a function of the viscosities of two liquids, drop size, and volume fraction. The results will be discussed in terms of internal circulation, drop deformation, and the breakup drops.

\section{EXPERIMENTAL}

\subsection{Composition and properties of emulsions}

For this study a series of oil-in-water emulsions was prepared. The oil was a mixture of $24 \mathrm{wt} \%$ tritolyphosphate and $76 \mathrm{wt} \%$ dioctylphthalate. The density and viscosity of the oil phase were $1.017 \times$ $10^{3} \mathrm{~kg} \cdot \mathrm{m}^{-3}$ and $6.54 \times 10^{-2} \mathrm{~Pa} \cdot \mathrm{s}$, respectively. The continuous phases were $20 \mathrm{wt} \%$ solutions of an anionic surfactant (Alipal CD-128 from GAF Corp.) in aqueous solutions of polyacrylic acid with a weight average molecular weight of $1.5 \times 10^{5}$ (Polysciences, Inc.). The polyacrylic acid was used to vary the viscosity of the aqueous phase.

Stock emulsions were prepared by gradually adding the oil to $20 \mathrm{wt} \%$ aqueous solution of surfactant while the solution was stirred gently with an impeller on a stirring motor. Since the desired volume fraction of dispersed phase is very high, careful attention must be paid to avoid entrapment of air bubbles and phase inversion. After the required amount of oil had been added, the emulsion was stirred at a constant mixing speed for $15 \mathrm{~min}$, so that all portions of the emulsion experienced approximately the same shear history. The drop size could be controlled by the final mixing speed. In this study, three stock emulsions with different drop sizes were made at a volume fraction of 0.97 . Figure 1 shows the drop size histograms in terms of number percentage. The drop size was determined from microphotographs of emulsions diluted with additional continuous phase. We calculated two types of mean diameters: the Sauter mean, $d_{\mathrm{sv}}$, and the root-mean-cube, $d_{\mathrm{rmc}}$, defined as

$$
d_{\mathrm{sv}}=\frac{\sum n_{\mathrm{i}} D_{\mathrm{i}}^{3}}{\sum n_{\mathrm{i}} D_{\mathrm{i}}^{2}}
$$

and

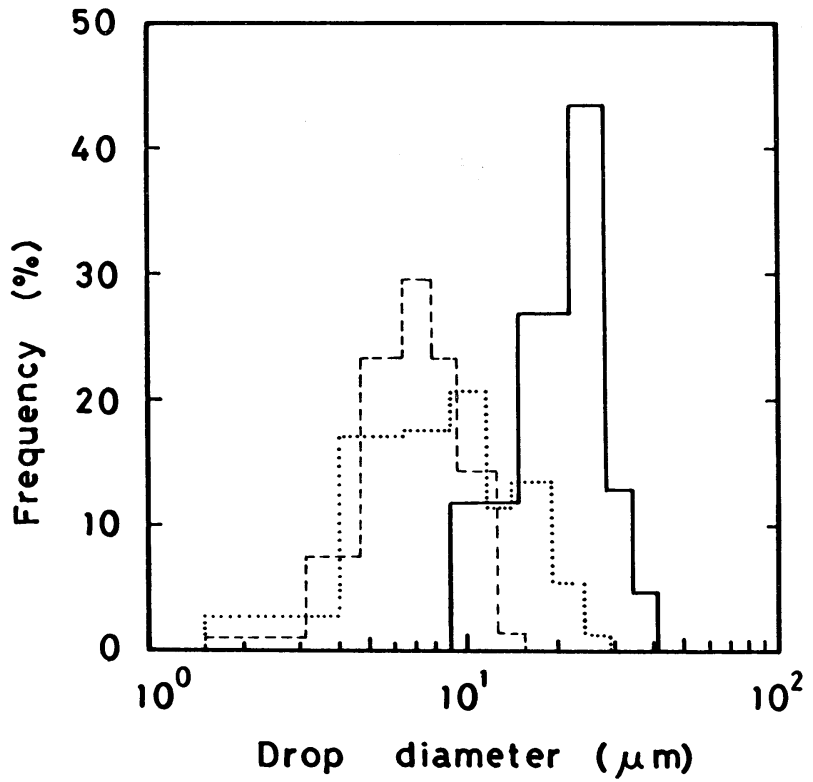

Fig. 1 Histograms of drop diameter in terms of number percentage: $(---)$; E1, (.....); E2, (-); E3.

Table I. Drop diameter.

\begin{tabular}{ccc}
\hline emulsion & $d_{\mathrm{sv}}(\mu \mathrm{m})$ & $d_{\mathrm{rmc}}(\mu \mathrm{m})$ \\
\hline E1 & 8.9 & 8.3 \\
E2 & 15.4 & 13.1 \\
E3 & 26.3 & 24.8 \\
\hline
\end{tabular}

$$
d_{\mathrm{rmc}}=\left(\frac{\sum n_{\mathrm{i}} D_{\mathrm{i}}^{3}}{\sum n_{\mathrm{i}}}\right)^{1 / 3}
$$

Here $n_{\mathbf{i}}$ is the number of drop with diameter $D_{\mathbf{i}}$. The size data are presented in Table I. The stock emulsions were stable for several months when stored in glass bottles.

Sample suspensions for rheological measurements were made by diluting the original stock emulsions with more of the continuous phase liquids. The volume fraction, $\Phi$, was from 0.1 to 0.9 . The dilution procedure was used to generate a series of four emulsions from a single stock emulsion. In each series, the emulsions have the same size distribution as the original one, but different continuous phase viscosities. This was accomplished by diluting the stock emulsions with calculated weights of aqueous solutions which contained polyacrylic acid in addition to $20 \mathrm{wt} \%$ surfactant. The final polymer concentrations in the continuous phase were $0,2.3$, 7.0 , and $19.0 \mathrm{wt} \%$. The properties of the continuous phase are given in Table II. All the solutions

Table II. Properties of continuous phase.

\begin{tabular}{ccccc}
\hline medium & $\begin{array}{c}\text { polymer } \\
\text { concentration } \\
(\mathrm{wt} \%)\end{array}$ & $\begin{array}{c}\text { density } \\
\rho \\
\left(\mathrm{kg} \cdot \mathrm{m}^{-3}\right)\end{array}$ & $\begin{array}{c}\text { viscosity } \\
\eta_{0} \\
(\mathrm{~Pa} \cdot \mathrm{s})\end{array}$ & $\begin{array}{c}\text { viscosity ratio } \\
\lambda\left(=\eta_{1} / \eta_{0}\right) \\
(-)\end{array}$ \\
\hline M1 & 0 & $1.009 \times 10^{3}$ & $2.65 \times 10^{-3}$ & 24.7 \\
M2 & 2.3 & $1.019 \times 10^{3}$ & $7.99 \times 10^{-3}$ & 8.19 \\
M3 & 7.0 & $1.030 \times 10^{3}$ & $5.37 \times 10^{-2}$ & 1.22 \\
M4 & 19.0 & $1.067 \times 10^{3}$ & $6.60 \times 10^{-1}$ & 0.098 \\
\hline
\end{tabular}


were Newtonian in the shear rate range of $1-250 \mathrm{~s}^{-1}$. The interfacial tension between oil and aqueous phases was measured by a spinning drop interfacial tensiometer. The obtained value is $3.2-3.4 \times$ $10^{-3} \mathrm{~N} \cdot \mathrm{m}^{-1}$ irrespective of polymer concentration.

\subsection{Rheological measurements}

The steady flow behavior was measured in the shear rate range of $0.1-250 \mathrm{~s}^{-1}$ using a parallel plate geometry on a Rheometrics System IV rheometer with a fluids transducer. Since the dispersed oil phase tends to coalesce at metal and plastic surfaces, the plates were made of glass disks whose diameter, $2 \mathrm{R}$, was $53.7 \mathrm{~mm}$. Concentrated emulsions and foams often slip at solid boundaries in rheometers and errors can arise in analyzing the dada. Yoshimura and Prud'homme ${ }^{22}$ have presented a technique of correcting for wall slip by repeating the same experiments twice in parallel plates with different gaps. When curves of shear stress versus apparent shear rate for different gaps coincide, there is no slip at the walls. To check the occurrence of wall slip, viscosity measurements were carried out at gap distances of 0.75 and $1.00 \mathrm{~mm}$ for each emulsion. Except for emulsions prepared with M4 medium, significant wall slip was not observed. Concentrated emulsions $(\Phi>0.7)$ in M4 medium were unstable under shear and a thick layer of oil was formed during measurements at the plate surfaces. As a result, the shear stress suddenly decreased at high shear rates. Therefore, the viscosity curve was determined in the shear rate range where the plots for different gaps lie on a single curve. Also, evaporation of aqueous phase causes coalescence of drops around the edge of upper disk, so that each run was completed within $30 \mathrm{~min}$.

\section{RESULTS}

Figure 2 shows the shear rate dependence of the viscosity for emulsions of oil drops with $d_{\mathrm{sv}}=8.9 \mu \mathrm{m}$ in the aqueous solution with $\eta_{0}=2.65 \times 10^{-3} \mathrm{~Pa} \cdot \mathrm{s}$ (these emulsions are labeled E1-M1). At volume fractions of $0.1-0.5$, the viscosity decreases with increasing shear rate and becomes constant above $100 \mathrm{~s}^{-1}$. The shear rate where the Newtonian flow begins is independent of volume fraction. When the volume fraction is increased beyond 0.5 , the emulsions show pseudoplastic behavior over the entire range of shear rates studied. A small increase in the volume fraction from $\Phi=0.6$ to 0.7 leads to a drastic increase in viscosity. Since the maximum random sphere packing is $\Phi_{\mathrm{c}}=0.64$, thin liquid films between drops are formed at concentrations above $\boldsymbol{\Phi}_{\mathbf{c}}$, leading to the large hydrodynamic forces.

Figure 3 shows the shear rate dependence of the viscosity for E2-M1 emulsions and Figure 4 for E3-M1 emulsions. Two important points are noted with regard to the effect of drop size. First, the Newtonian viscosity is constant irrespective of drop size, although it increases with volume fraction. Second, the viscosity increases with decreasing drop size in the pseudoplastic region. Since the critical shear rate above which the flow of emulsions $(\Phi<$ 0.5 ) is Newtonian decreases with increasing drop size, an increase of drop size causes a horizontal

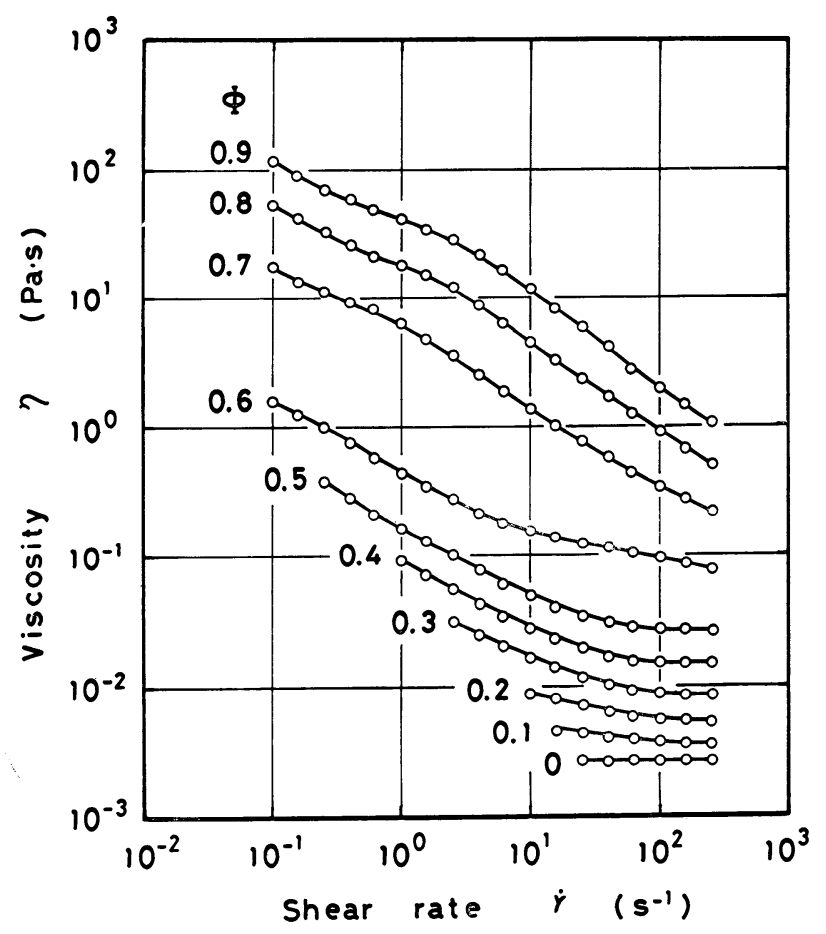

Fig. 2 Shear rate dependence of viscosity for E1M1 emulsions.

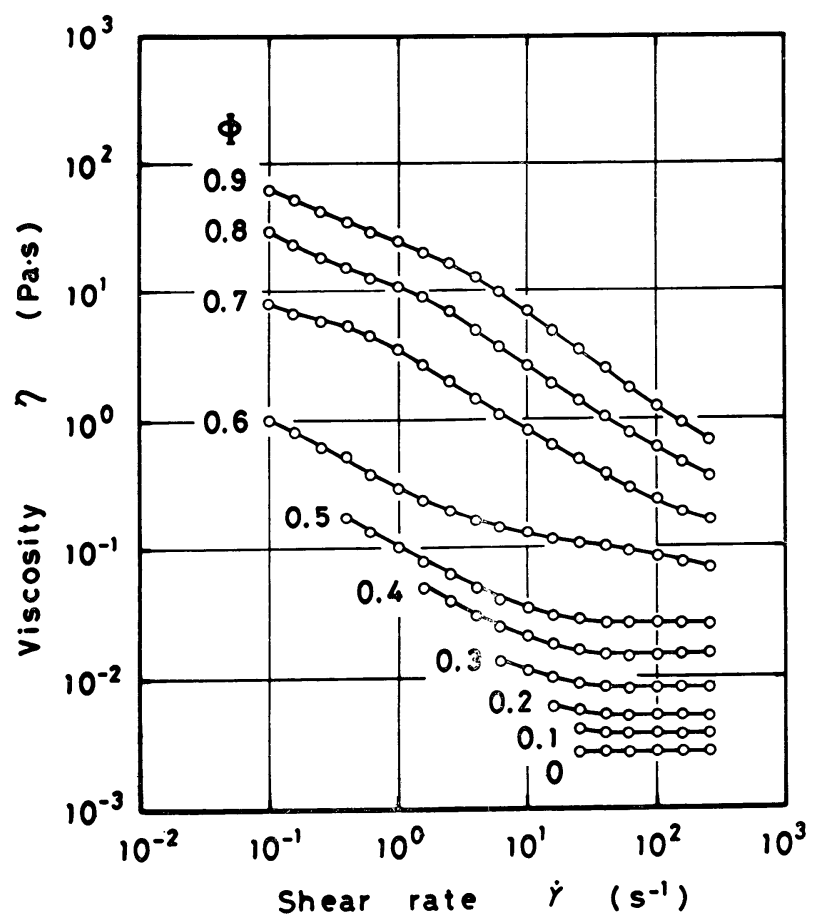

Fig. 3 Shear rate dependence of viscosity for E2M1 emulsions.

shift of the viscosity curve toward lower shear rates.

Figure 5 shows the effect of drop size on the viscosity behavior for emulsions dispersed in M2 medium. Since the viscosity of the aqueous phase is increased, the emulsions in M2 medium have 


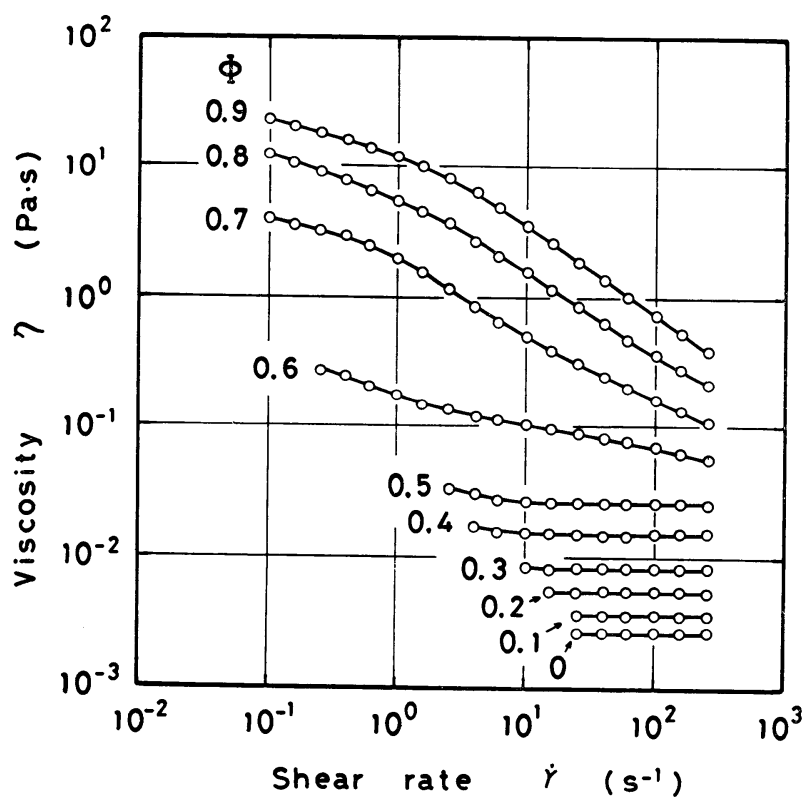

Fig. 4 Shear rate dependence of viscosity for E3M1 emulsions.

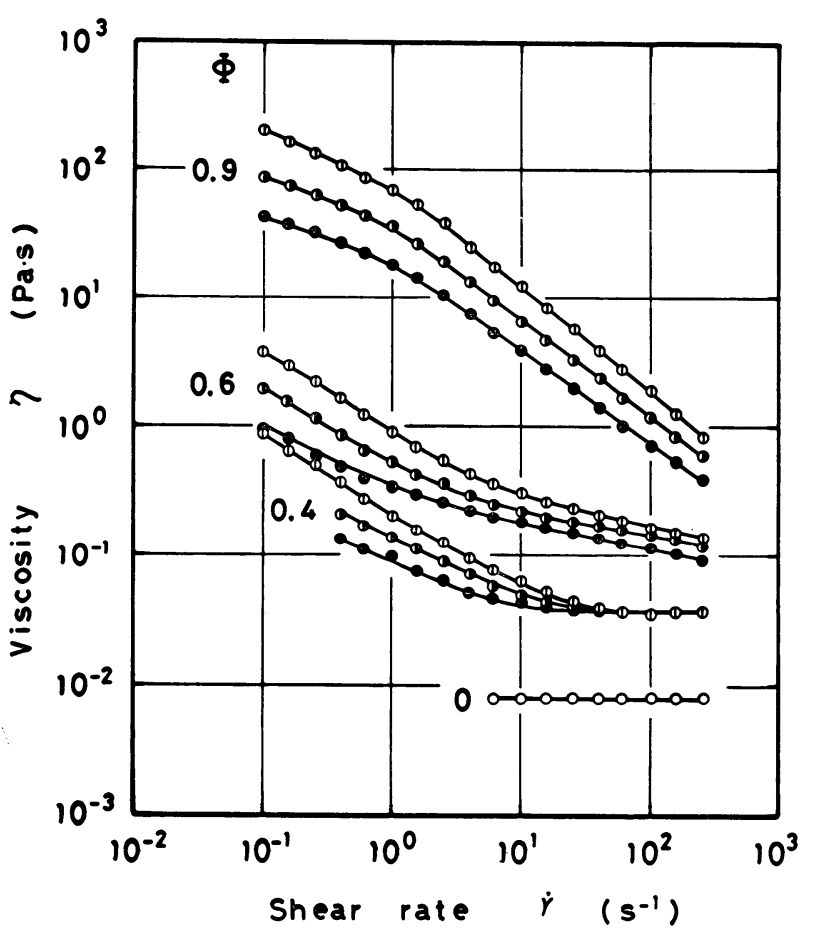

Fig. 5 Effect of drop size on the viscosity behavior for emulsions dispersed in M2 medium: (1); E1, ((); E2, (๑); E3.

higher viscosities than those in Ml medium. The emulsions with $\Phi=0.4$ are also Newtonian at high shear rates and the Newtonian viscosity is independent of drop size. In the pseudoplastic region, the viscosity increases with decreasing drop size. Although the M2 medium has a viscosity three times higher than the Ml medium, the effect of aqueous phase viscosity becomes weak at high volume fractions as is predicted by the theories of

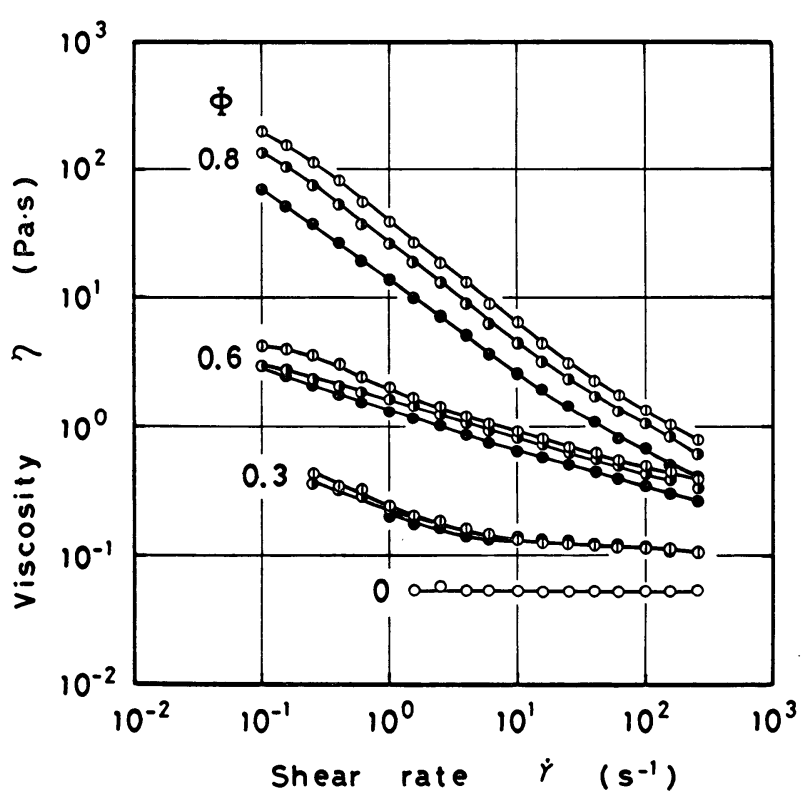

Fig. 6 Effect of drop size on the viscosity behavior for emulsions dispersed in M3 medium: (Ф); E1, ((); E2, (๑); E3.

cellular materials.

Figure 6 shows the effect of drop size on the viscosity behavior for emulsions in M3 medium. The aqueous phase of these emulsions has a viscosity comparable to the oil phase. At $\Phi=0.3$, the emulsion shows Newtonian behavior at high shear rates, but the relative viscosities at equal volume fraction are not as large as those of emulsions in M1 medium. When the volume fraction exceeds 0.4 , the highshear-rate Newtonian region disappears and the flow turns pseudoplastic. It is of interest to note that with increasing viscosity of the aqueous phase, the slope of viscosity curve decreases at low shear rates for emulsion at $\Phi=0.4$. Although the emulsions with smaller drops show higher viscosity, the size effect becomes weak with decreasing viscosity ratio.

Figure 7 shows the effect of drop size for emulsions in the high viscosity M4 medium. When the continuous phase is much more viscous than the dispersed phase, the viscosity augmentation is suppressed at high shear rates. Especially at $250 \mathrm{~s}^{-1}$, the viscosities of the emulsions are all of the same order while the low-shear-rate viscosities vary by 3 orders of magnitude. Even at low volume fraction of $\Phi=0.3$, the emulsion is pseudoplastic. The drop size has no significant effect on the viscosity behavior at volume fractions less than $\Phi=0.4$. The emulsions with $\Phi=0.6$ show Newtonian flow at low shear rates. But this Newtonian viscosity varies with drop size. The viscosity rapidly falls at high shear rates. After viscosity measurements, concentrated emulsions were often covered with a lubricating layer of oil indicating that the emulsions were unstable under shear and drops coalesced at the surface.

The viscosity behavior of emulsions are summarized as follows:

(a) At low volume fractions, the viscosity de- 


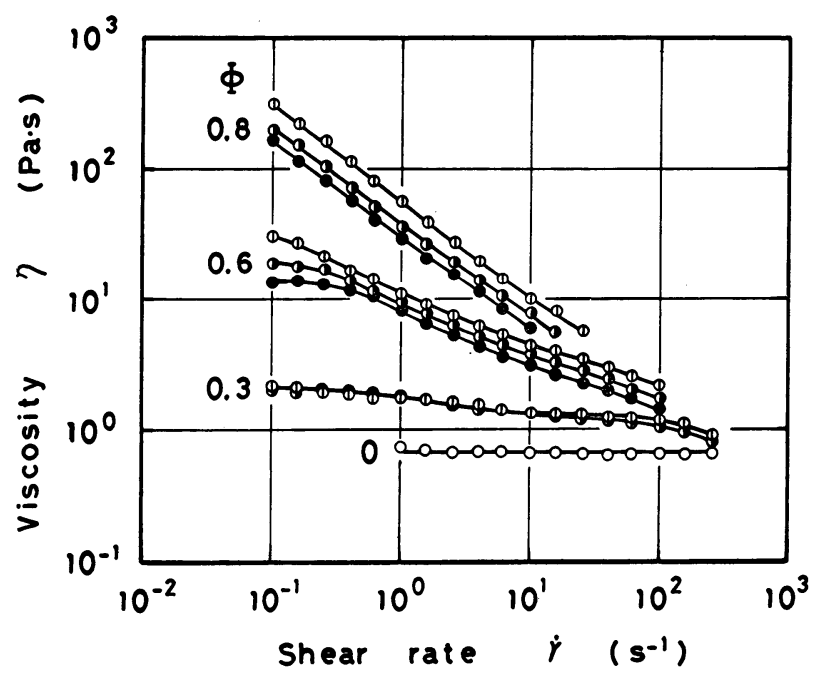

Fig. 7 Effect of drop size on the vsicosity behavior for emulsions dispersed in M4 medium: ((1); E1, (O); E2, (O); E3.

creases with increasing shear rate and becomes constant at high shear rates. The Newtonian viscosity is independent of drop size.

(b) At high volume fractions, the emulsions show pseudoplastic flow over the entire range of shear rates from 0.1 to $250 \mathrm{~s}^{-1}$. The viscosity in the pseudoplastic region increases with decreasing drop size.

(c) When the viscosity ratio of the internal to external phases decreases, the viscosity augmentation (relative viscosity) at a given volume fraction is reduced and the dependence of viscosity on the drop size becomes weak. In addition, emulsions with low viscosity ratio and with volume fractions below $\Phi=0.5$ show pseudoplastic flow at high shear rates while Newtonian flow is observed for emulsions with large viscosity ratio.

\section{DISGUSSION}

According to Einstein's classical theory of suspension rheology, the increase in viscosity is due to the distortion of the velocity field in the vicinity of each particle. If the dispersed particles are fluid drops, the tangential stresses across the interface between the continuous and dispersed phases cause internal circulation which results in a reduction of distortion of the flow field outside the drops. Taylor ${ }^{9)}$ showed that at a velocity gradient low enough for the drops to remain spherical, the viscosity of an emulsion is given by

$$
\begin{aligned}
& \frac{\eta}{\eta_{0}}=1+K \Phi \\
& K=\frac{5}{2} \frac{0.4+\lambda}{1+\lambda}
\end{aligned}
$$

where $\eta$ and $\eta_{0}$ are the viscosities of the emulsion and continuous phase, respectively, $\lambda$ is the viscosity ratio of the internal to external phases $\left(=\eta_{\mathrm{i}} / \eta_{0}\right)$, and $K$ is designated as the Taylor coefficient which varies from 1 to 2.5 by changing $\lambda$ from 0 to $\infty$.
For suspensions of solid particles, one empirical equation that takes into account the effect of higher dispersed phase concentrations is the Mooney equation $^{23)}$. Provided that there is no interference between the effect of particle interactions and internal circulation, the equation may be extended to moderately concentrated emulsions.

$$
\frac{\eta}{\eta_{0}}=\exp \left(\frac{K \Phi}{1-\Phi}\right)
$$

Eq. (5) implies that a plot of $\ln \left(\eta / \eta_{0}\right)$ versus $\Phi /(1-\Phi)$ gives a straight line with the slope being the Taylor coefficient. However, the Taylor and Mooney theories assume that the viscosities of disperse systems are Newtonian. Therefore, we analyzed the Newtonian viscosity $\eta_{\mathrm{N}}$ at high shear rates.

Figure 8 shows the relation between $\ln \left(\eta_{\mathrm{N}} / \eta_{0}\right)$ and $\Phi /(1-\Phi)$. The plot for each system gives a fairly straight line whose slope decreases with decreasing viscosity ratio. Since the Newtonian viscosity is independent of drop size as mentioned above, the slope is only a function of viscosity ratio. Table III shows the comparison of the theoretical prediction with the experimental results for the Taylor coefficient. Good agreement between them suggests that the drops remain spherical and the internal circulation is fully developed inside the drops. Previous studies $^{24)}$,25) have measured values of $K$ larger than those predicted by Taylor theory. This may be due to partial circulation inside the drops in those experiments, resulting from surfactant concentration gradients on the drop surface that retard internal circulation. Oldroyd ${ }^{26)}$,27) extended Taylor's equation by introducing the two-dimensional shear viscosity and area viscosity of film. These two effects can increase the Taylor coefficient. In our case, the Taylor coefficients are closely fitted by eq. (4)

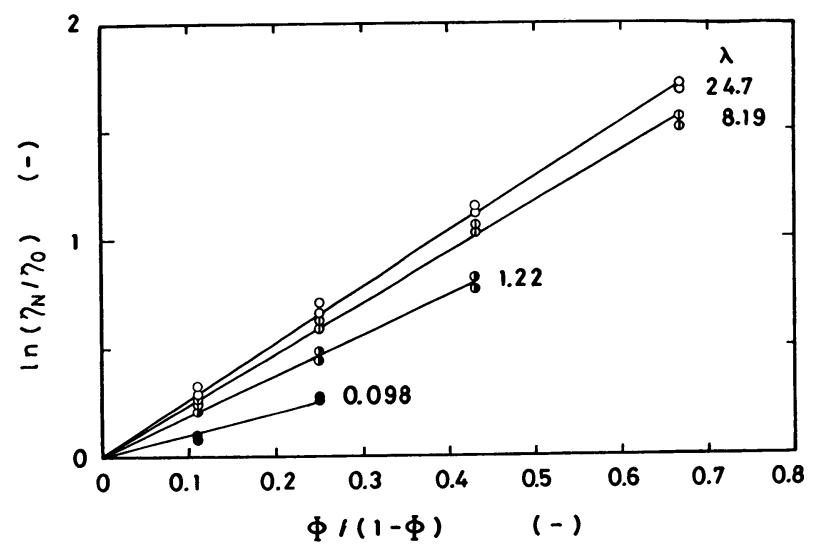

Fig. 8 Logarithmic plots of relative viscosity against $\Phi /(1-\Phi)$.

Table III. Taylor coefficient $K$.

\begin{tabular}{ccc}
\hline $\begin{array}{c}\text { Viscosity ratio } \\
\lambda\end{array}$ & $K_{\text {cal }}$ & $K_{\text {exp }}$ \\
\hline 24.7 & 2.44 & 2.55 \\
8.19 & 2.34 & 2.35 \\
1.22 & 1.82 & 1.86 \\
0.098 & 1.13 & 1.08 \\
\hline
\end{tabular}


because of high surfactant concentration and low interfacial tension.

In suspensions of non-interacting particles (such as sterically stabilized suspensions), the balance between Brownian motion and hydrodynamic forces governs the dynamic structure under shear flow. The suspension viscosity is scaled by the Péclet number $P_{\mathbf{e}}\left(=\eta_{0} \dot{\gamma} a^{3} / k T\right)$, where $a$ is the particle radius and $k T$ the thermal energy ${ }^{28)}$. Willey and Macosko ${ }^{29)}$, and Krieger ${ }^{30), 31)}$ have observed that the relative viscosities measured using different particle radii and fluid viscosities are superimposed when plotted against the Péclet number. Since the drops of emulsions used in this study are treated as spheres in the Newtonian range, we have examined the applicability of superposition and shift factors. Figure 9 shows the master curves at $\Phi=0.4$ for all of the emulsions studied. The data have been superimposed by a horizontal shift along the shear rate axis (relative to the data for the E1 emulsion). It can be seen that for the same viscosity ratio the viscosity behavior for emulsions with different drop sizes superimposes very well. Figure 10 shows the shift factor plotted

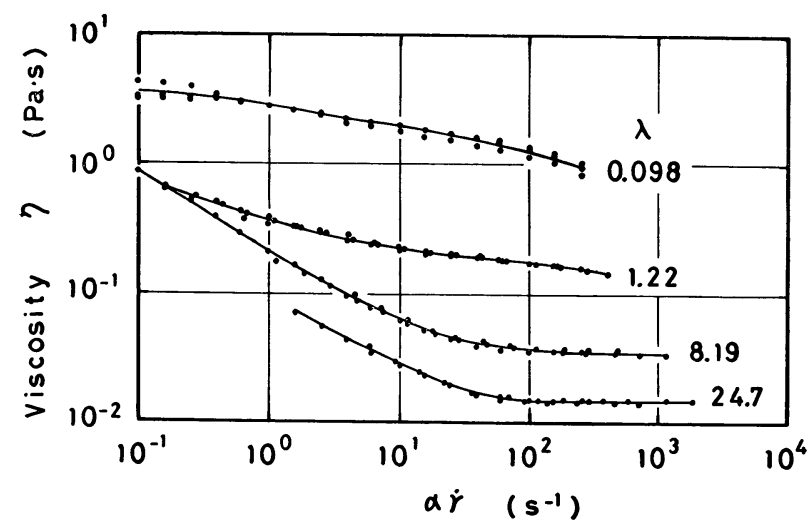

Fig. 9 Master curves at $\Phi=0.4$ reduced to $\mathrm{E} 1$ emulsion.

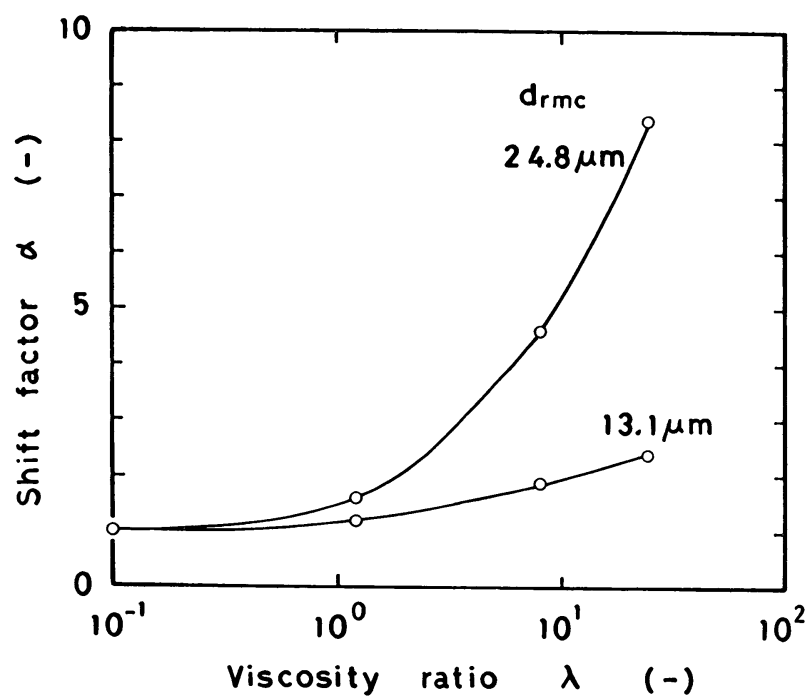

Fig. 10 Shift factor plotted against viscosity ratio of internal to external phases. against the viscosity ratio. For volume fraction below 0.5 , the shift factor does not vary with volume fraction. There are two important findings from the superposition of the viscosity curves. First, the shift factor decreases with decreasing viscosity ratio, whereas in sterically stabilized suspensions, it is independent of the viscosity of the medium for a constant particle size. At the lowest viscosity ratio the difference in drop size causes no shift in the viscosity curve. At the highest viscosity ratio, where the drops appear to behave as hard spheres with a Taylor coefficient almost the same as the Einstein coefficient $(K=2.5)$, there is a strong dependence of the shift factor on drop size. However, cubed power dependence on particle radius observed for Brownian hard-sphere suspensions is not observed. Rather squared dependence on size is observed that would be expected from a direct scaling on the hydrodynamic force $\left(6 \pi \eta_{0} a^{2} \dot{\gamma}\right)$ for these non-Brownian particles. Also, the appropriate average diameter to be used is the root-mean-cube diameter, which is related to particle volume, rather than the Sauter mean diameter, which is related to particle surface area.

The second point is that four master curves in Fig. 9 can not be superimposed. Different shapes of the viscosity curves imply that the drop dynamics is not scaled by the viscosity ratio. The most interesting feature of the original viscosity curves is that the high-shear-rate Newtonian range disappears at low viscosity ratios. The deformation of the drops is responsible for this pseudoplastic behavior at high shear rates. The deformation of drop is characterized by a deformation parameter $D$ involving the length $L$ and $B$ of the major and minor axes of the elliptical drop:

$$
D=\frac{L-B}{L+B}
$$

Taylor's theory ${ }^{32)}$ for deformation of single isolated drop is used here to estimate the deformation of drops.

$$
D=\frac{\eta_{0} \dot{\gamma} a}{\sigma} \frac{19 \lambda+16}{16 \lambda+16}=C_{\mathrm{a}} \frac{19 \lambda+16}{16 \lambda+16}
$$

Here, $\sigma$ is the interfacial tension and $C_{\mathrm{a}}$ the Capillary number. From eq. (7) the deformation parameter is 0.06 for E3-M3 emulsion at $250 \mathrm{~s}^{-1}\left(\alpha \dot{\gamma}=400 \mathrm{~s}^{-1}\right.$ in Fig. 9). If the viscosity of the emulsion, $\eta$, is used instead of the aqueous phase viscosity, $\eta_{0}$, the deformation parameter is 0.14 . In general, the drop is regarded as deformed when the deformation parameter exceeds 0.05 . It is concluded that the pseudoplastic behavior at high shear rates is attributed to deformation of drops.

Similarly, the superposition has been attempted for emulsions above $\Phi=0.7$. Figure 11 shows the results for three different volume fractions. Although the data slightly scatter, a reasonably good superposition is obtained. The three curves lie very close at high shear rates. In this region, the deformation parameter increases to 0.5 and above. The theoretical prediction of the critical deformation parameter, above which a drop will be broken by shear forces, is $D=0.5$. Since the coalescence and breakup 


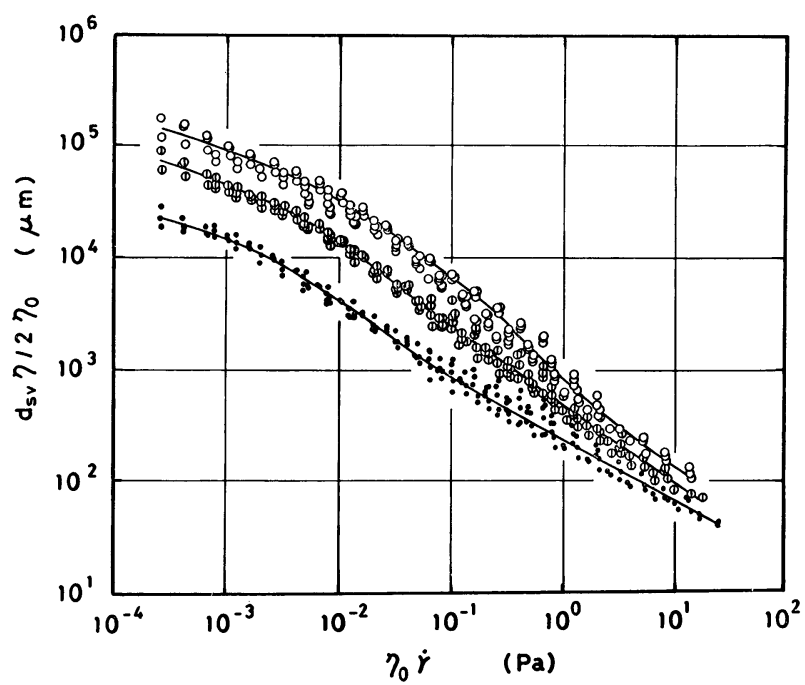

Fig. 11 Master curves for highly concentrated emulsions: $(\odot) ; \Phi=0.7, \quad(Ф), \Phi=0.8$, $(\bigcirc) ; \Phi=0.9$.

of drops are induced at the same time by shear forces, it is not possible to relate bulk rheology to drop size in the high-shear-rate regime.

A point to be noted is that the change in drop size causes a vertical shift of viscosity curves in contrast to emulsions less than $\Phi=0.6$. In highly concentrated emulsions, drops can not remain spherical, and a thin liquid film is formed between two deformed drops. When the emulsion is subjected to shear forces, the liquid film must stretch or shrink until a critical strain is achieved that results in steady flow. The stress corresponding to this critical state gives the yield stress. Therefore, the elasticity of liquid films plays an important role in controlling the rheology. Because the total interfacial area between two liquids is inversely proportional to Sauter mean diameter, $d_{\mathrm{sv}}$, the viscosity curves for emulsions at different drop sizes are superimposed by the vertical shift. Also, increases in interfacial tension should lead to similar vertical shifts. Since the stresses during flow for concentrated emulsions arise from elastic stresses in the interstitial films, it is of interest to study the viscoelastic properties above the critical volume fraction.

\section{References}

1) Princen HM, J Colloid Interface Sci, 91, 160 (1983).

2) Princen HM, J Colloid Interface Sci, 105, 150 (1985).

3) Princen HM, Kiss AD, J Colloid Interface Sci, 112, 427 (1986).

4) Prud'homme RK, Paper presented at the 53rd Annual Meeting of the Society of Rheology, Lexington, Kentucky, Oct. 11-15, 1981.

5) Kraynik AM, Hansen MG, J Rheol, 30, 409 (1986).

6) Khan SA, Armstrong RC, J Non-Neretonian Fluid Mech, 22, 1 (1986).

7) Khan SA, Armstrong RC, J Non-Neretonian Fluid Mech, 25, 61 (1987).

8) Khan SA, Schnepper CA, Armstrong RG, J Rheol, 32, 69 (1988).

9) Taylor GI, Proc Roy Ser A, 138, 41 (1932).

10) Rumscheidt FD, Mason SG, J Colloid Sci, 16, 210 (1961).

11) Flumerfelt RW, J Colloid Interface Sci, 76, 330 (1980).

12) Phillips WJ, Graves RW, Flumerfelt RW, J Colloid Interface Sci, 76, 350 (1980).

13) Frankel NA, Acrivos A, J Fluid Mech, 44, 65 (1970).

14) Choi SJ, Schowalter WR, Phys Fluids, 18, 420 (1975).

15) Vadas EB, Goldsmith HL, Mason SG, Trans Soc Rheol, 20, 373 (1976).

16) Bagley EB, Christiansen DD, Beckwith AC, J Rheol, 27, 503 (1983).

17) Bredimas M, Veyssie M, J Non-Nerwtonian Fluid Mech, 12, 165 (1983).

18) Jayasuriya DS, Varanasi PP, Tchenrekdjian N, Colloid Surfaces, 21, 371 (1986)

19) Lissant KJ, Mayhan KG, J Colloid Interface Sci, 42, 201 (1973).

20) Lissant KJ, Peace BW, Mayhan KG, J Colloid Interface Sci, 47, 416 (1974)

21) Princen HM, Aronson MP, Moser JC, J Colloid Interface Sci, 75, 246 (1980).

22) Yoshimura A, Prud'homme RK, J Rheol, 32, 53 (1988).

23) Mooney M, J Colloid Sci, 6, 162 (1951).

24) Nawab MA, Mason SG, Trans Faraday Soc, 54, 1712 (1958).

25) Barnea E, Mizrahl J, Ind Eng Chem Fundam, 15, 120 (1976).

26) Oldroyd JG, Proc Roy Soc Ser A, 218, 122 (1953).

27) Oldroyd JG, Proc Roy Soc Ser A, 232, 567 (1955).

28) Krieger IM, Trans Soc Rheol, 7, 101 (1963).

29) Willey SJ, Macosko CW, J Rheol, 22, 525 (1978).

30) Krieger IM, Adv Colloid Interface Sci, 3, 111 (1972).

31) Krieger IM, Choi GN, "Advances in Rheology", Vol. 2, Mena B, Garcia-Rejon A, Rangel-Nafaile C, ed, (1984), Universidad National Autonoma de Mescices, Mexico City, p. 641.

32) Taylor GI, Proc Roy Soc Ser A, 146, 501 (1934). 